\section{AUTOIMMUNITY}

\section{Gene therapy for diabetes}

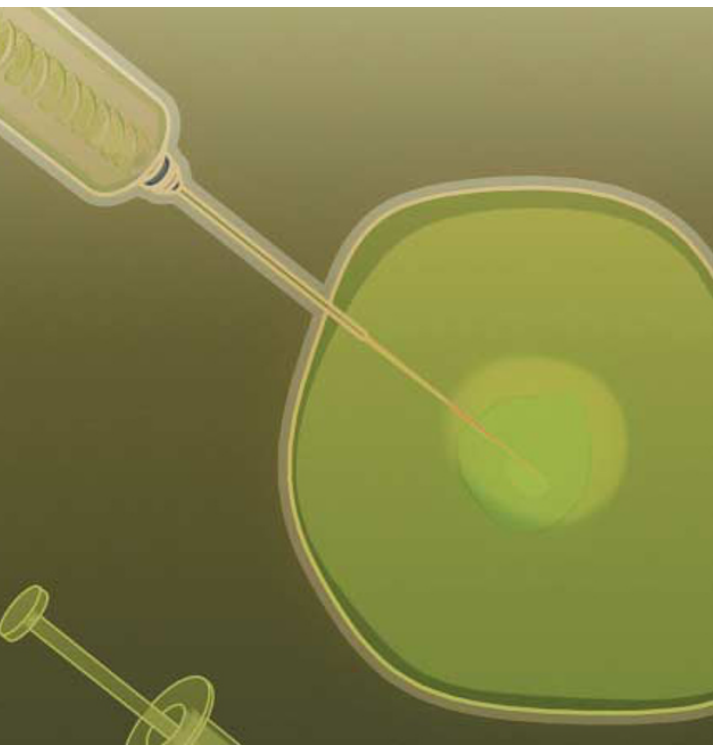

Type 1 diabetes mellitus (T1DM) occurs as a result of T-cell-mediated destruction of the insulin-producing $\beta$-cells in the pancreas. Non-obese diabetic (NOD) mice, which spontaneously develop diabetes, are a useful model system of this disease. In this study, the authors show that expression of diabetes-resistant MHC class II alleles by NOD mice, using retroviral transduction of autologous bone-marrow cells, is sufficient to prevent the development of diabetes.

In humans, the development of T1DM is associated with the inheritance of particular MHC class II alleles that lack a charged amino acid at position 57 of the $\beta$-chain. In NOD mice, the single MHC class II allele present is $\mathrm{I}-\mathrm{A}^{\mathrm{g} 7}$, which also lacks a charged residue at position 57. It is thought that lack of a charged residue at this position prevents the formation of a salt bridge between the $\alpha$ - and $\beta$-chains of the MHC class II molecule, which could affect the ability of these molecules to mediate negative selection of autoreactive T cells.

Previous studies using transgenic mice and allogeneic bone-marrow chimeras have shown that it is possible to prevent diabetes in NOD mice, but it has not been possible to determine the preventative mechanism.
Retroviral constructs with green fluorescent protein fused to the cytoplasmic tails of diabetesresistant I-A $\beta$-chains (which have a charged residue at position 57) were shown to be expressed at the cell surface of MHC-class-IIpositive cells and could pair with endogenous $\alpha$-chains. Young NOD mice were lethally irradiated and transplanted with bone marrow retrovirally transduced with the $\mathrm{I}-\mathrm{A} \beta$ constructs, and their blood-glucose levels were monitored each week. All of the mice were protected from diabetes, compared with four of six mice that received bone marrow transfected with a control construct. Even when diabetes was aggressively induced using cyclophosphamide, the mice that received $\mathrm{I}-\mathrm{A} \beta$-transduced bone marrow were resistant to the development of diabetes for up to 46 weeks after transfer, and the level of insulitis was reduced.

To address the mechanism of resistance, the authors used enzyme-linked immunosorbent spot (ELISPOT) assays to look at the frequency of $\mathrm{T}$ cells that produced cytokines in response to peptide 206-220 from glutamic-acid decarboxylase, an immunodominant self-antigen peptide in NOD mice. No cytokine production was detectable in NOD mice that received

\title{
Memories are made of this...
}

Infection with the parasite Leishmania causes considerable morbidity and mortality, against which there is no effective human vaccine. Both humans and mice can resolve primary infections and become resistant to further infection, but some parasites persist and might contribute to long-term protection by maintaining the presence of effector T cells. If persistent antigen is required for long-term protection, then developing a non-live vaccine against leishmaniasis will be difficult. In this study, Phillip Scott and colleagues characterized the $\mathrm{CD}^{+} \mathrm{T}$-cell response during infection and showed that protective central memory $\mathrm{T}\left(\mathrm{T}_{\mathrm{CM}}\right)$ cells, which are not dependent on the presence of parasites, can develop in infected mice.

Memory T cells are a heterogeneous population thought to contain two distinct subsets - effector memory $\mathrm{T}\left(\mathrm{T}_{\mathrm{EFF}}\right)$ cells, which migrate to tissues and produce cytokines, and $\mathrm{T}_{\mathrm{CM}}$ cells, which circulate through the lymph nodes. Scott and colleagues investigated the development of $\mathrm{CD}^{+}$ memory $\mathrm{T}$ cells during infection of mice with Leishmania major. $\mathrm{CD}^{+} \mathrm{T}$ cells were labelled with CFSE (5,6-carboxyfluorescein diacetate succinimidyl ester) — which allows proliferative responses to be analysed by flow cytometry - and then transferred into naive recipients. After parasitic challenge infection of the recipient mice, some of these donor $\mathrm{T}$ cells migrated to the draining lymph nodes (dLNs) and proliferated, indicating that immune mice contain a $\mathrm{T}_{\mathrm{CM}}$-cell population. During proliferation, most cells downregulated their expression of the lymphnode homing molecule CD62L, indicating that these cells had differentiated into $\mathrm{T}_{\mathrm{EFF}}$ cells. To confirm that $\mathrm{T}_{\mathrm{CM}}$ cells present in the donor T-cell population from immune mice could differentiate into $T_{\mathrm{EFF}}$ cells and mediate protection, $\mathrm{CD} 4{ }^{+} \mathrm{CD} 62 \mathrm{~L}^{\text {hi }} \mathrm{T}$ cells which do not produce interferon- $\gamma($ IFN $-\gamma)$ when stimulated with leishmanial antigens were purified and used in transfer experiments. Again, the donor T cells proliferated in the dLN; they also developed the capacity to produce IFN- $\gamma$ and could be detected at the site of infection within two weeks. Importantly, the mice that received these $T_{C M}$ cells were protected from challenge infection.
To address the role of persistent antigen in the maintenance of memory $\mathrm{T}$ cells, a mutant $L$. major strain that is unable to persist in mice was used. Mice infected with this mutant $L$. major had no detectable parasites by 15 weeks after infection. At 25 weeks after infection, no $T_{\mathrm{EFF}}$ cells were detectable, as shown by the lack of both an IFN- $\gamma$ response to leishmanial antigens in vitro and a delayed-type hypersensitivity response in vivo. However, $\mathrm{T}_{\mathrm{CM}}$ cells were detectable at 25 weeks; CFSE-labelled CD4 ${ }^{+}$ $T$ cells from these mice, when transferred to naive recipients that were subsequently challenged with $L$. major, could migrate to and proliferate in the dLN. Furthermore, 25 weeks after the initial infection with mutant $L$. major, mice were protected against infection with virulent $L$. major, showing that the $\mathrm{T}_{\mathrm{CM}}$ cells conferred protection.

This study shows that both $\mathrm{T}_{\mathrm{EFF}}$ and $\mathrm{T}_{\mathrm{CM}}$ cells contribute to immunity to L. major infection, but $\mathrm{T}_{\mathrm{CM}}$ cells can be maintained in the absence of parasites and confer protection. Targeting $\mathrm{T}_{\mathrm{CM}}$ cells could therefore be the basis for the development of a successful non-live vaccine against leishmaniasis.

Elaine Bell

(2) References and links ORIGINAL RESEARCH PAPER Zaph, C., Uzonna, J. Beverley, S. M. \& Scott, P. Central memory T cells mediate long-term immunity to Leishmania major in the absence of persistent parasites. Nature Med. 10, 1104-1110 (2004). 
